$1-1-2007$

\title{
Excess radiation from the large planets
}

Richard L. Liboff

University of Central Florida

Find similar works at: https://stars.library.ucf.edu/facultybib2000 University of Central Florida Libraries http://library.ucf.edu

This Article is brought to you for free and open access by the Faculty Bibliography at STARS. It has been accepted for inclusion in Faculty Bibliography 2000s by an authorized administrator of STARS. For more information, please contactSTARS@ucf.edu.

\section{Recommended Citation}

Liboff, Richard L., "Excess radiation from the large planets" (2007). Faculty Bibliography 2000s. 7354.

https://stars.library.ucf.edu/facultybib2000/7354

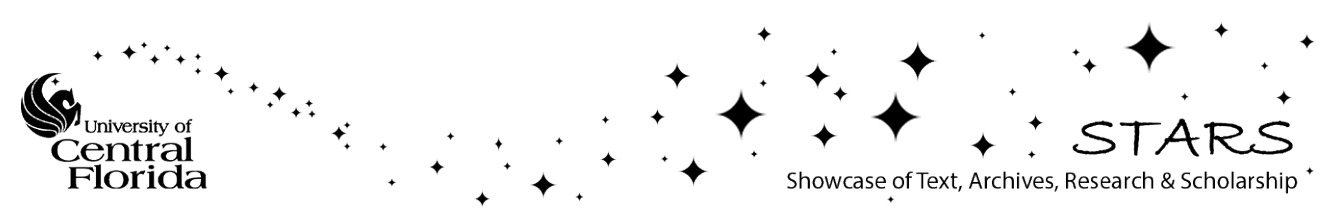


The Astronomical Journal, 134:2179-2185, 2007 December

(C) 2007. The American Astronomical Society. All rights reserved. Printed in U.S.A.

\title{
EXCESS RADIATION FROM THE LARGE PLANETS
}

\author{
RichARD L. LIBOFF \\ Physics Department, University of Central Florida, Orlando, FL 32816-2385, USA; rll@physics.ucf.edu \\ Received 2005 July 28; accepted 2007 July 15
}

\begin{abstract}
An alternative model is proposed for the excess radiation emitted by the larger planets, each with a liquid metallic hydrogen annular domain about a central core of ice and rocks. This model is based on the mutual attraction between elements of an aggregate of charged bosons in a charge-neutralizing background, in equilibrium at very high pressure, and the property that spin-1 deuterons are bosons. Assuming valid parameters for Jupiter, it is derived that the deuteron density in Saturn is approximately equal to that in Jupiter and that particles emitted in reactions in the liquid metal domain are thermalized in the liquid hydrogen domain, resulting in infrared radiation, in accord with observed values. With corroborating properties of Neptune, it is proposed that this planet likewise contains a spherical shell of liquid metallic hydrogen outside and close to its rocky core. Whereas data are insufficient to support degenerate fusion, the known magnetic moment of Neptune is found to be consistent with positive charge components rotating in the frame of the liquid metallic hydrogen fluid with current density $\approx 8.4 \times 10^{-6} \mathrm{~A} \mathrm{~m}^{-2}$. It is proposed that the related coupling between current and magnetic field is supported by a dynamo effect. A brief description is included describing the influence of convective storms in the large planets.
\end{abstract}

Key words: magnetic fields — planets and satellites: general — radiation mechanisms: general

\section{INTRODUCTION}

In this work an alternative model is proposed for the anomalous radiation property of larger planets Jupiter and Saturn of emitting more radiant energy than they receive from the Sun. This model derives from the liquid metallic hydrogen component of these planets. As first proposed by Salpeter \& Stevenson (1977a), an aggregate of hydrogen molecules will break up into an electrically conductive mixture of protons and electrons at a pressure greater than $3 \times 10^{6} \mathrm{~atm}$, values typical of the central domains of these large planets. Models that have been proposed in the past to explain this excess radiative emission are as follows: accretion of matter during planet formation (de Pater \& Lissauer 2001), release of gravitational energy due to planet contraction and/or helium sedimentation (Salpeter \& Stevenson 1977b; Guillot 1999), decay of radioactive isotopes in the core (Hubbard 1989), and deuteron burning (Coraddu et al. 2002). Due to related properties of Neptune, which in like manner emits infrared radiation in excess of that received by the Sun, and the fact that the thermal evolution model for generated internal heat fails for Neptune (de Pater \& Lissauer 2001), it is proposed that this planet likewise includes a spherical shell of liquid metallic hydrogen outside and close to its rocky core. An estimate of the dipole magnetic moment of Neptune stemming from this proposed liquid metallic core corroborates this hypothesis.

It is generally stated that the excess radiation emitted by these planets does not have its origin in nuclear processes. Thus, for example, for Jupiter it is argued that the planet does not have sufficient mass to support gravitationally induced fusion (Baugher 1990). The fusion process in the present model does not derive from gravitational attraction and related high-energy collisions. It is based on the property that in a frame rotating with the planet, the liquid metallic hydrogen state is in a thermodynamic equilibrium phase at a given pressure and temperature, and that in this condition charged bosons exist in a charge-neutralizing background. (The radiative emission of these planets is an infinitesimally small perturbation to the respective equilibrium state.)
Consequently, components of these bosons attract one another. That is, the radial distribution function for the charged bosons does not vanish at zero displacement (Leung et al. 1976; Foldy 1961; Feenberg 1969; Girardeau 1962; Liboff 1979). We recall that deuterons, with spin 1, are bosons. A closely allied property is that an equilibrium aggregate of noninteracting bosons will exhibit a statistical attractive potential (Huang 1987). In another related study it was shown that in the ground state of a collection of interacting deuterons in a steady magnetic field, spins are polarized in the direction of the field (Liboff 1994). In the present work it is argued that fusion of deuterons takes place in the stable liquid phase of metallic deuterium of these planets that is estimated to exist in the thermodynamic region, $P \geq 10^{6}$ bars, $T \geq$ $10^{4} \mathrm{~K}$. A list of temperature increments between the expected planetary temperature from solar incidence and that measured is presented, for which Jupiter has the largest incremental value and Uranus has the smallest value.

In a related work by Ouyed et al. (1998), excess heat in the Jovian planets was calculated due to high-energy deuteron Coulomb interactions with deuterons, tritons, and helium-3 nuclei. A work more closely allied with that of the present study is that of Kitamura (2000). In this study nuclear reactions were examined in the highdensity limit, $r_{s}=\left(m e^{2} a_{e} / \hbar^{2}\right)=\left(m e^{2} / \hbar^{2}\right)\left(3 / 4 \pi n_{e}\right)^{1 / 3} \ll 1$, where $a_{e} \equiv\left(3 / 4 \pi n_{e}\right)^{1 / 3}$ is the Wigner-Seitz radius and $n_{e}$ is the electron density ("pycnonuclear" reactions). (We recall that $r_{s}$ represents the ratio of $a_{e}$ to the Bohr radius, $a_{0}=\hbar / m e^{2}$, where $m$ is the electron mass.) It was found that nuclear reaction rates are proportional to $\Gamma=\exp \left(-C \rho_{m}^{-1 / 6}\right)$, where $C$ is a constant and $\rho_{m}$ is the mass density. Calculations were derived from Monte Carlo data on screened potentials. Whereas the present work likewise addresses the domain $r_{s} \ll 1$, as noted, it is based on the property that an equilibrium medium of charged bosons in a neutralizing background and very high pressure includes a radial distribution function that is finite at the origin (no displacement between deuterons). The process is not related to standard fusion derived from high-energy collisions. Furthermore, no previous formulation has employed the Bose property of deuterons in studies of the excess radiation emitted by the Jovian planets. 


\section{ANALYSIS}

Let $\Gamma$ represent the ratio of emitted radiant energy flux $I_{e}$ to incident radiant energy flux $I_{o}$ of a planet, so that

$$
\Gamma=I_{e} / I_{o}
$$

For Jupiter the temperature in the liquid metallic hydrogen shell is $T \simeq 19,000^{\circ} \mathrm{C}$, and

$$
\Gamma_{\mathrm{J}} \simeq 1.67
$$

with radiation emitted (at a wavelength in the range $10-100 \mu \mathrm{m}$ ) in the infrared spectrum. The pressure near the center of the planet is $P \simeq 3.6 \times 10^{7} \mathrm{~atm}$. In absolute magnitude Jupiter radiates at

$$
W_{\mathrm{J}}=4 \times 10^{17} \mathrm{~W} \text {. }
$$

In Saturn this value is

$$
W_{\mathrm{S}}=2 \times 10^{17} \mathrm{~W}
$$

(Morrison \& Owen 1987).

The temperature in the liquid metallic domain in Saturn is $T \simeq 15,000^{\circ} \mathrm{C}$. The pressure in this region is $P \simeq 5.0 \times 10^{7} \mathrm{~atm}$, and

$$
\Gamma_{\mathrm{S}} \simeq 3
$$

The ice-rock core density is $\rho_{\mathrm{S}} \simeq 19 \mathrm{~g} \mathrm{~cm}^{-3}$ (Baugher 1990).

The proton number density in Jupiter is (Coraddu et al. 2002)

$$
n_{\mathrm{J}}(p)=2.4 \times 10^{24} \mathrm{~cm}^{-3} \text {. }
$$

This value is based on the core mass density, $\rho_{\mathrm{J}}=5 \mathrm{~g} \mathrm{~cm}^{-3}$, and $k_{\mathrm{B}} T=2 \mathrm{eV}\left(1 \mathrm{eV}=11,605 \mathrm{~K}=1.602 \times 10^{-19} \mathrm{~J}\right)$. The deuteronto-proton number-density ratio in the interior of this planet is

$$
n_{\mathrm{J}}(d) \simeq 3 \times 10^{-5} n_{\mathrm{J}}(p) .
$$

It has been noted that observational determination of deuteron densities in these planets has not been established (Guillot 1999). In the present study, cited values for this parameter for Jupiter (Coraddu et al. 2002) are assumed to be appropriate. Theoretical estimates on the $d / p$ ratio in these planets (Mahaffy et al. 1998) indicate a slightly greater value in Saturn than in Jupiter. In the present work, we use Jupiter data to obtain a value of $n_{\mathrm{S}}(p)$ for Saturn, which is found to be approximately equal to that of $n_{\mathrm{J}}(p)$.

The total gravitational and centrifugal force on a deuteron in the metallic phase of hydrogen is given by

$$
F_{d}=M_{d}\left(-\frac{G M_{\mathrm{J}}}{r^{2}}+\omega^{2} r\right)=r M_{d}\left[-G\left(\frac{4 \pi}{3}\right) \rho_{\mathrm{J}}+\omega^{2}\right] \text {, }
$$

where $M_{d}$ and $M_{\mathrm{J}}$ are the deuteron and Jupiter masses, respectively, $G$ is the gravitational force constant, $\rho_{\mathrm{J}}$ is the mass density of Jupiter, and $\omega$ is the angular frequency of the planet. In the preceding relations the gravitational force far exceeds the centrifugal force, which may, in turn, be omitted. A parallel equation applies for the proton component of the fluid. If the proton mass is $m$, then $M_{d} \simeq 2 m$, and we may conclude that the "fall" toward the central domain of the planet is greater for deuterons than for protons. We note that this simple example neglects other electric and fluid forces. However, as a first approximation, one may conclude that the force (eq. [5c]) contributes to a separation of the deuteron and proton components of the fluid. As noted, in a frame rotating with the planet, this fluid component is taken to be in a thermodynamic equilibrium state. In this configuration the stated separation is maintained with no distortion due to diffusion.

The fusion reaction in the present model derives from the fact that fermion protons in the fluid repel one another, whereas Bose deuterons at the given thermodynamic conditions attract one another.

\subsection{Fusion Parameters}

The relevant fusion reactions in this formulation are

$$
\begin{gathered}
d+d \rightarrow \mathrm{He}^{3}(0.82)+n(2.84) \\
d+d \rightarrow t(1.01)+p(3.02)
\end{gathered}
$$

Subsidiary reactions are

$$
\begin{gathered}
5 \mu \text { barns, } p+t \rightarrow \mathrm{He}^{3}+\gamma+5.5 \mathrm{MeV}, \\
7 \mu \text { barns, } \alpha+\gamma+19.8 \mathrm{MeV}
\end{gathered}
$$

where $\alpha, d, n$, and $t$ represent alpha, deuteron, neutron, and triton particles, respectively, and in equations (6a) and (6b) the parenthetical numbers represent decay-product energies in $\mathrm{MeV}$. The primary $(d, d)$ reactions occur at cross section $\sigma \simeq 0.6$ barn, where 1 barn $=10^{-24} \mathrm{~cm}^{2}$. The subsidiary reactions (eqs. [6c] and [6d]) occur at microbarn levels and may be neglected in the formulation. The triton decay product in equation (6b) has a half-life of $12.26 \mathrm{yr}$ and decays as follows:

$$
t \rightarrow \beta+\mathrm{He}^{3},
$$

in which the beta particle is emitted with an energy of $18 \mathrm{keV}$.

The mean radii of displacement (in AU; $1 \mathrm{AU}=1.496 \times$ $10^{8} \mathrm{~km}$ ) from the Sun for these two planets are

$$
R_{\mathrm{J}} \simeq 5.2, \quad R_{\mathrm{S}} \simeq 9.54 .
$$

The mean radiation flux emitted by the Sun is

$$
I_{o}=6.44 \times 10^{7} \mathrm{~W} \mathrm{~m}^{-2} \text {. }
$$

The radiant fluxes falling on these planets are, respectively,

$$
I_{\mathrm{J}}=I_{o}\left(\frac{r_{\sigma}}{R_{\mathrm{J}}}\right)^{2}, \quad I_{\mathrm{S}}=I_{o}\left(\frac{r_{\sigma}}{R_{\mathrm{S}}}\right)^{2},
$$

where

$$
r_{\sigma} \simeq 6.96 \times 10^{5} \mathrm{~km}=4.65 \times 10^{-3} \mathrm{AU}
$$

is the radius of the Sun. The equatorial radius of Jupiter is

$$
R_{\mathrm{J}} \simeq 71,400 \mathrm{~km}=7.14 \times 10^{7} \mathrm{~m} .
$$

The radial increment of the liquid metal annular domain in Jupiter is (Baugher 1990; Friedlander 1985; Morrison \& Owen 1987)

$$
R_{\mathrm{J}}^{(p)} \simeq 45,600 \mathrm{~km}=4.56 \times 10^{7} \mathrm{~m}
$$

outside of a core of rock and ice with radius

$$
R_{\mathrm{J}}^{(c)} \simeq 13,400 \mathrm{~km}=1.34 \times 10^{7} \mathrm{~m} .
$$


The radius to the outer limit of the liquid metal section is

$$
R_{\mathrm{J}}^{(p+c)}=5.90 \times 10^{7} \mathrm{~m} .
$$

The parallel relations for Saturn are

$$
R_{\mathrm{S}} \simeq 60,000 \mathrm{~km}=6.00 \times 10^{7} \mathrm{~m} .
$$

The ice-rock core of Saturn is of radius

$$
R_{\mathrm{S}}^{(c)} \simeq 16,000 \mathrm{~km}=1.60 \times 10^{7} \mathrm{~m}
$$

whereas the annular liquid metal domain has the incremental radius

$$
R_{\mathrm{S}}^{(p)} \simeq 14,000 \mathrm{~km}=1.40 \times 10^{7} \mathrm{~m}
$$

The radius to the outer limit of the liquid metal section is

$$
R_{\mathrm{S}}^{(p+c)}=3.0 \times 10^{7} \mathrm{~m} .
$$

Respective volumes of liquid metal domains in these planets are

$$
\begin{gathered}
V_{\mathrm{J}}^{(p)}=\frac{4}{3} \pi\left[\left(R_{\mathrm{J}}^{(p+c)}\right)^{3}-\left(R_{\mathrm{J}}^{(c)}\right)^{3}\right] \approx \frac{4}{3} \pi\left(\alpha_{\mathrm{J}} R_{\mathrm{J}}^{(p+c)}\right)^{3} \\
V_{\mathrm{S}}^{(p)}=\frac{4}{3} \pi\left[\left(R_{\mathrm{S}}^{(p+c)}\right)^{3}-\left(R_{\mathrm{S}}^{(c)}\right)^{3}\right] \approx \frac{4}{3} \pi\left(\alpha_{\mathrm{S}} R_{\mathrm{S}}^{(p+c)}\right)^{3}
\end{gathered}
$$

where

$$
R_{\mathrm{J}}^{(p+c)}=R_{\mathrm{J}}^{(p)}+R_{\mathrm{J}}^{(c)}=5.9 \times 10^{7} \mathrm{~m}
$$

The presence of protons in the liquid metal domain reduces the effective $d$ - $d$ fusion volume. With equation (5b) we note that $n_{\mathrm{J}}(d) \simeq 10^{-5} n_{\mathrm{J}}(p)$. The $d$ - $d$ interaction is further moderated by shielding (Liboff 1958). These effects are incorporated in the $\alpha$-factor in equations (12a) and (12b), and we write $\alpha \simeq 10^{-8}$. Due to the statistical attraction of deuterons in the present configuration, the cross section of the $d$ - $d$ interaction is moderated. For energies ( $\lesssim 1 \mathrm{keV})$, charged particle cross sections vanish. This property is incorporated in the Wigner exponential factor (Blatt \& Weisskopf 1952) that vanishes in the limit. In the present configuration, due to statistical attraction of deuterons, we take the $d-d$ cross section to be given by the square of the cross-sectional area of the nucleus. Accordingly, we take the nuclear diameter to be $2.3 \times 10^{-13} \mathrm{~cm}^{-1}$, which gives the $d$ - $d$ cross section, $\sigma \approx$ $5.3 \times 10^{-32} \mathrm{~m}^{-2}$. The primary $d$ - $d$ interaction in relation (6) has the yield $f \approx 6 \mathrm{MeV}$.

An estimate of the net radiation emitted per second from Jupiter is given by

$$
W_{\mathrm{J}} \simeq \sigma f v V_{\mathrm{J}}^{(p)} n_{\mathrm{J}}^{2}(d) \mathrm{W}
$$

where $v$ is the deuteron speed, given by (Coraddu et al. 2002)

$$
v=\sqrt{\frac{2 E}{M}} \simeq 1.38 \times 10^{4} \mathrm{~m} \mathrm{~s}^{-1}
$$

and $E$ is the deuteron energy. We obtain

$$
W_{\mathrm{J}} \simeq 3.12 \times 10^{14} \mathrm{~W}
$$

in partial agreement with measured values.

\section{QUANTUM DOMAIN AND RADIAL DISTRIBUTION FUNCTION}

We consider an aggregate of $N$ charged bosons confined to the volume $V$ with mean number density $n$ in a neutralizing background. The radial distribution function $g(r)$ is related to the pair probability function $p_{2}(1,2)$ as (Goodstein 1975)

$$
p_{2}(1,2)=n^{2} g(r)
$$

where $r$ is the corresponding scalar displacement between the two particles. The pair probability function has the normalization

$$
V \int p_{2}(1,2) d \boldsymbol{r}=N(N-1) .
$$

The criterion to determine whether the medium, at the temperature $T$, is in the quantum domain is given by comparing the thermal de Broglie wavelength, $\lambda_{d}$, to the mean interparticle spacing, $r_{d}=n_{\mathrm{J}}^{-1 / 3}(d)$, where

$$
\lambda_{d}=\frac{h}{\left(2 \pi m k_{\mathrm{B}} T\right)^{1 / 2}} .
$$

Namely, if

$$
\lambda_{d} \gtrsim r_{d}
$$

quantum mechanics is relevant. With equation $(14 \mathrm{~b})$ we obtain $r_{d} \simeq 2.4 \times 10^{-7} \mathrm{~cm}$. At $k_{\mathrm{B}} T \simeq 2 \mathrm{eV}$, we find for the related deuteron medium $\lambda_{d}=0.81 \times 10^{-7} \mathrm{~cm}$. It follows that the liquid metallic components of these planets are in the quantum domain.

The Hamiltonian of our system, in second quantization, is given by

$$
\begin{aligned}
H\left(\boldsymbol{k}^{N}, \boldsymbol{S}^{N}\right)= & \sum_{\boldsymbol{k}} t_{k} a_{\boldsymbol{k}}^{\dagger} a_{\boldsymbol{k}}+\frac{1}{2 V} \sum_{\boldsymbol{k}^{\prime \prime}} \sum_{\boldsymbol{k}^{\prime}}^{\prime} \sum_{\boldsymbol{k}} g_{k} a_{\boldsymbol{k}^{\prime \prime}-\boldsymbol{k}}^{\dagger} a_{\boldsymbol{k}^{\prime}+\boldsymbol{k}}^{\dagger} a_{\boldsymbol{k}^{\prime \prime}} a_{\boldsymbol{k}^{\prime}} \\
& -\tau\left(\frac{\mu_{d}}{2 \hbar}\right)^{2} \sum_{i \neq j} \boldsymbol{S}_{i} \cdot \boldsymbol{S}_{j}
\end{aligned}
$$

where

$$
t_{k}=\hbar^{2} k^{2} / 2 M, \quad g_{k}=4 \pi^{2} / k^{2}
$$

$a_{k}^{\dagger}$ and $a_{k}$ are creation and annihilation operators, respectively, for particles of momentum $\hbar \boldsymbol{k}$ that satisfy standard boson commutation relations, $M$ is the boson mass, $\mu_{d} \simeq 10^{-23}$ ergs $\mathrm{G}$, and the constant $\tau$ has dimensions $\mathrm{G}^{2} \mathrm{~cm}^{3}$ and in part reflects the short range of the spin-spin interaction. The prime on the second sum in the middle term of equation (15a) indicates that the term with $k=0$ is omitted, which accounts for the neutralizing background charge. In the present study effects of spin are neglected.

We wish to obtain an expression for the relative number of particles in the ground state of the system. To these ends we recall the Bogoliubov transformation (Bogoliubov 1947) that is based on the following assumptions: (1) Eliminate terms in the second set of sums in equation (15a) that contain fewer than two creation or annihilation operators for particles of momentum zero. (2) Replace $a_{0}^{\dagger}$ and $a_{0}$ in the remaining terms by the $c$ number $N_{0}^{1 / 2}$, where $N_{0} \gg 1$ is the mean occupation number of the ground state. Note 
that this latter Bogoliubov criterion implies that total number of particles is not conserved. In general, one may write

$$
N=N_{0}+\left\langle\bar{N}_{0}\right\rangle
$$

where $\left\langle\bar{N}_{0}\right\rangle$ is the expectation of the number of bosons in states other than the ground state. With these assumptions, the Hamiltonian (eq. [15a]) reduces to

$$
H\left(\boldsymbol{k}^{N}\right)=\sum_{k}^{\prime}\left[\left(t_{k}+n_{0} g_{k}\right) a_{\boldsymbol{k}}^{\dagger} a_{\boldsymbol{k}}+\frac{1}{2} n_{0} g_{k}\left(a_{\boldsymbol{k}} a_{-\boldsymbol{k}}+a_{\boldsymbol{k}}^{\dagger} a_{-\boldsymbol{k}}^{\dagger}\right)\right],
$$

where

$$
n_{0}=N_{0} / V
$$

The Hamiltonian (eq. [16b]) is diagonalized by the canonical transformation

$$
a_{k}=S_{k+} b_{k}-S_{k-} b_{-k}^{\dagger}
$$

where

$$
\begin{gathered}
S_{\boldsymbol{k}( \pm)}^{2}=t_{k} n_{0} g_{k} \mp \epsilon_{k} / 2 \epsilon_{k}, \\
\epsilon_{k}^{2}=\hbar^{2} \omega_{p}^{2}+\left(\hbar^{2} k^{2} / 2 M\right)^{2} \\
\omega_{p}^{2} \equiv 4 \pi n_{0} e^{2} / M
\end{gathered}
$$

The parameter $\omega_{p}$ is the boson plasma frequency. The Hamiltonian (eq. [16b]) is then given by the diagonal form

$$
\begin{gathered}
H=U_{0}+\sum_{\boldsymbol{k}}^{\prime} \epsilon_{k} b_{\boldsymbol{k}}^{\dagger} b_{\boldsymbol{k}}, \\
U_{0} \equiv \sum_{\boldsymbol{k}}^{\prime} S_{\boldsymbol{k}}^{2},
\end{gathered}
$$

where $S_{k}$ is written for $S_{k-}$. In the limit that $N \propto \Omega \rightarrow \infty$, the sum (eq. [18b]) becomes

$$
U_{0}=\frac{\Omega}{2 \pi^{2}} \int_{0}^{\infty} S_{k}^{2} d k
$$

The ratio of bosons not in the ground state to those in the ground state of the system is given by

$$
\frac{N-N_{0}}{N_{0}}=\frac{1}{2 \pi^{2} n_{0}} \int_{0}^{\infty} S_{k}^{2} d k .
$$

We introduce the new variable of integration,

$$
\xi=\left(\frac{a_{b}}{4 \pi n_{0}}\right)^{1 / 4} k
$$

where

$$
a_{b}=\frac{\hbar^{2}}{M e^{2}}=a_{0} \frac{m}{M} \ll a_{0},
$$

in which $a_{0}=0.5292 \AA$ is the hydrogen Bohr radius and $a_{b} \approx$ $1.44 \times 10^{-12} \mathrm{~cm}$ is the effective deuteron Bohr radius. The ground state of a deuteron is composed of $S$ and $D$ states, with respective probability densities of 0.96 and 0.04 , so that a deuteron is highly spherical. However, the $D$ state causes a slight extension of the nuclear wave function, and we take the effective value of $a_{b} \approx$ $10^{-11} \mathrm{~cm}$. The neutralizing background introduces a shielding which further defines the core of the deuteron. In the standard model of the ground state of the deuteron (Burcham 1963) the predominant component of the wave function lies outside the core and decays exponentially. With these properties we write $a_{b} \approx 1.44 \times 10^{-10} \mathrm{~cm}$.

The ratio (eq. [19a]) may be written

$$
\frac{\alpha}{4} \equiv \frac{N-N_{0}}{N_{0}}=Q r_{s}
$$

where the parameter $\alpha$ is familiar to this area of study (de Pater \& Lissauer 2001; Leung et al. 1976; Morrison \& Cruikshank 1981),

$$
r_{s}=\left(\frac{3}{4 \pi}\right)^{1 / 3} \frac{1}{a_{b} n_{0}^{1 / 3}}
$$

and the integral $Q$ has the value (de Pater $\&$ Lissauer 2001)

$$
Q=\left(\frac{1}{3 \pi^{4}}\right)^{1 / 4} \int\left[\frac{\xi^{4}+2}{\left(\xi^{4}+4\right)^{1 / 2}}-\xi^{2}\right] d \xi=0.2114
$$

It is noted that for Coulomb systems such as in the present case (de Pater \& Lissauer 2001; Leung et al. 1976),

$$
\alpha=4 Q r_{s}=1-g(0)
$$

where $g(r)$ is the radial distribution function (Liboff 2003) for the interboson displacement, $r$. It follows that for sufficiently small $Q r_{s}$, there is wave-function overlap at the origin resulting in fusion from either of the two reactions (eqs. [6a] and [6b]). Thus, our criterion for fusion in the present configuration is that $\alpha \ll 1$, or, equivalently, that

$$
a_{b} n_{0}^{1 / 3} \gg 0.2624
$$

With equation (19b) and the stated assumptions, we write $a_{b}=$ $1.43 \times 10^{-10} \mathrm{~cm}$ so that criterion (21a) becomes

$$
n_{0} \equiv N_{0} / N \gg 3.5 \times 10^{30} \mathrm{~cm}^{3}
$$

which is noted to likewise satisfy the Bogoliubov criterion $n_{0} \gg 1$. With equation (5b), $n_{\mathrm{J}} \simeq 7.2 \times 10^{19} \mathrm{~cm}^{-3}$, so that $\gamma \equiv n_{\mathrm{J}} / n_{0} \simeq$ $10^{-11}$. The radial distribution is a statistical entity. Namely, we recall that the factor $g(\boldsymbol{r}) d \boldsymbol{r} / V$ represents the probability of finding a pair of deuterons with one particle at the origin and the other at $\boldsymbol{r}+d \boldsymbol{r}$ in the volume element $d \boldsymbol{r}$. The accessible volume is $V$. We surmise that a fraction $\gamma$ of deuterons in Jupiter will fuse by this mechanism.

\section{DEUTERON DENSITY IN SATURN}

With the result (eq. [21b]) at hand, we turn to Saturn. It is noted that $W_{\mathrm{J}} \propto V_{\mathrm{J}}^{(p)} n_{\mathrm{J}}^{2}(d)$, with the remaining parameters assumed constant. The result is

$$
W_{\mathrm{S}} \simeq W_{\mathrm{J}} \frac{V_{\mathrm{S}}^{(p)} n_{\mathrm{S}}^{2}(d)}{V_{\mathrm{J}}^{(p)} n_{\mathrm{J}}^{2}(d)}
$$


Here we assume that the $d / p$ ratios in Jupiter and Saturn are approximately equal. With the cited value (Morrison \& Owen 1987), $W_{\mathrm{S}}=2 \times 10^{17} \mathrm{~W}$, there follows $W_{\mathrm{S}} / W_{\mathrm{J}} \simeq 1 / 2$, and we find

$$
n_{\mathrm{S}}^{2}(d) \simeq \frac{W_{\mathrm{S}}}{W_{\mathrm{J}}} \frac{V_{\mathrm{J}}^{(p)}}{V_{\mathrm{S}}^{(p)}} n_{\mathrm{J}}^{2}(d) \simeq \frac{1}{2}\left(\frac{5.9}{3}\right)^{3} n_{\mathrm{J}}^{2}(d) \simeq 3.8 n_{\mathrm{J}}^{2}(d)
$$

which gives the first estimate,

$$
n_{\mathrm{S}}(d) \simeq 2 n_{\mathrm{J}}(d)
$$

\subsection{Centrifugal and Density Effects}

Relation (22c) may be further modified due to centrifugal and density effects. The equatorial period of rotation of Saturn is $\tau_{\mathrm{S}} \simeq 10^{\mathrm{h}} 14^{\mathrm{m}}$, and in Jupiter it is $\tau_{\mathrm{J}} \simeq 9^{\mathrm{h}} 50^{\mathrm{m}}$ (Baugher 1990 ; Friedlander 1985). The tangential speed of the boundary of the corresponding metallic domain is

$$
v_{\mathrm{S}}=\frac{R_{\mathrm{S}}^{(p+c)}}{\tau_{\mathrm{S}}} \simeq \frac{2 \pi 30,000 \mathrm{~km}}{3.68 \times 10^{4} \mathrm{~s}}=4.91 \mathrm{~km} \mathrm{~s}^{-1} .
$$

The corresponding value in Jupiter is

$$
v_{\mathrm{J}}=\frac{R_{\mathrm{J}}^{(p+c)}}{\tau_{\mathrm{J}}} \simeq \frac{2 \pi 59,000 \mathrm{~km}}{3.54 \times 10^{4} \mathrm{~s}}=10.47 \mathrm{~km} \mathrm{~s}^{-1}
$$

It follows that the centrifugal separation force in Saturn is less than that in Jupiter. With the preceding, this effect on the deuteron density in Saturn is to reduce it by the factor

$$
\frac{v_{\mathrm{S}}^{2}}{v_{\mathrm{J}}^{2}} \frac{R_{\mathrm{J}}^{(p)}}{R_{\mathrm{S}}^{(p)}} \simeq 0.927
$$

The effects of metallic core densities may be further included by noting that the densities of these planets are due largely to the core masses. In Jupiter the mean density is $\rho_{\mathrm{J}} \simeq 1.3 \mathrm{~g} \mathrm{~cm}^{-3}$, and in Saturn it is $\rho_{\mathrm{S}} \simeq 0.7 \mathrm{~g} \mathrm{~cm}^{-3}$. It follows that the relative deuteron density in Saturn is further modified by the factor $\rho_{\mathrm{S}} / \rho_{\mathrm{J}} \simeq 0.54$. Combining these two effects gives

$$
n_{\mathrm{S}} \simeq 0.927 \times 0.54 \times 2 n_{\mathrm{J}} \simeq 1.00 n_{\mathrm{J}},
$$

so that the deuteron density in Saturn is approximately equal to that in Jupiter.

\subsection{Temperature Increments}

The excess infrared radiation emitted by these planets is inferred by the increment between measured temperature $T_{\text {meas }}$ and expected temperature $T_{\text {exp }}$, derived from solar incidence. We set

$$
T_{\text {meas }}=T_{\exp }+\Delta T \text {. }
$$

For Jupiter, one obtains (Baugher 1990)

$T_{\text {meas }}^{(\mathrm{J})}=-149^{\circ} \mathrm{C}, \quad T_{\exp }^{(\mathrm{J})}=-165^{\circ} \mathrm{C}, \quad \Delta T^{(\mathrm{J})}=16^{\circ} \mathrm{C}$.

For Saturn,

$T_{\text {meas }}^{(\mathrm{S})}=-179^{\circ} \mathrm{C}, \quad T_{\exp }^{(\mathrm{S})}=-190^{\circ} \mathrm{C}, \quad \Delta T^{(\mathrm{S})}=11^{\circ} \mathrm{C}$.
For Neptune,

$T_{\text {meas }}^{(\mathrm{N})}=-218^{\circ} \mathrm{C}, \quad T_{\exp }^{(\mathrm{N})}=-227^{\circ} \mathrm{C}, \quad \Delta T^{(\mathrm{N})}=9^{\circ} \mathrm{C}$.

In each case $T_{\text {meas }}$ is higher (i.e., less negative) than $T_{\text {exp }}$, indicating an internal energy source in the corresponding planet.

\section{NEPTUNE AND URANUS}

Neptune, like Jupiter and Saturn, radiates energy in excess of that received by the Sun, with relatively smaller radiative output,

$$
\begin{gathered}
W_{\mathrm{N}}=3 \times 10^{13} \mathrm{~W}, \\
\Gamma_{\mathrm{N}} \gtrsim 2
\end{gathered}
$$

(Moore 2000). These four planets (including Uranus) are the most massive in the solar system. (In Earth masses, $M_{\mathrm{J}}=318$, $M_{\mathrm{S}}=95.1, M_{\mathrm{N}}=17.2$, and $M_{\mathrm{U}}=14.5$.) We wish to ascertain whether Neptune contains a liquid hydrogen internal component. Regarding Uranus, for which $\Delta T^{(\mathrm{U})} \simeq 0.1^{\circ} \mathrm{C}$ (de Pater \& Lissauer 2001), it is noted that any internal energy source of this planet must be exceedingly small, outside the limits of Voyager instrumentation. That is, $\Gamma_{\mathrm{U}} \lesssim 1.06$ with $94 \%$ error (Baugher 1990; Moore 2000).

The equatorial radius of Neptune is

$$
R_{\mathrm{N}} \simeq 24,800 \mathrm{~km}
$$

A plausible model of the structure of this planet (Friedlander 1985) includes a central core composed of rock and metal of radius

$$
R_{\mathrm{N}}^{(c)} \simeq 8000 \mathrm{~km}
$$

with an exterior domain composed of methane, ammonia, and ice beneath a proposed shell of liquid molecular hydrogen. Modeling the planet after Jupiter gives the incremental radius of the proposed liquid component as

$$
R_{\mathrm{N}}^{(p)} \simeq 2.72 \times 10^{4} \mathrm{~km}
$$

The average density of the planet is $1.67 \mathrm{~g} \mathrm{~cm}^{-3}$. The pressure at the central core is $P \simeq 2.2 \times 10^{7}$ atm at a temperature of $7000^{\circ} \mathrm{C}$, and the equatorial period of rotation is $\simeq 0.75-1.0$ Earth days. It follows that this planet satisfies the criteria for deuteron fusion, save for the existence of a liquid metal hydrogen component. Suppose that this component exists. We estimate the related magnetic field of Neptune and compare it to the measured values. For a bounded collection of $N$ identical particles, each with an angular momentum component $\boldsymbol{L}_{i}$ and a charge-to-mass ratio $q / M$, the magnetic moment is given by (Jackson 1999)

$$
\mathcal{M}=\frac{q}{2 M} \sum_{i=1}^{N} \boldsymbol{L}_{i}=\frac{q}{2 M} \boldsymbol{L} .
$$

We recall that the angular momentum of a system with a moment of inertia $I$ and rotating with mean angular frequency $\omega$ is given by $L \simeq I \omega$. For a spherical system of radius $R, I=(2 / 5) R^{2}$. We compare the measured and estimated ratios of the magnetic moments of Neptune to Jupiter. In terms of Earth's magnetic 
moment $($ Baugher 1990$), \mathcal{M}_{\mathrm{N}} \simeq 2 \mathcal{M}_{\mathrm{E}}$ and $\mathcal{M}_{\mathrm{J}} \simeq 3000 \mathcal{M}_{\mathrm{E}}$, so that the measured ratio is

$$
\mathcal{M}_{\mathrm{N}} / \mathcal{M}_{\mathrm{J}} \simeq 6.7 \times 10^{-4}
$$

A rough estimate of this ratio is given by the following. First, we note the rotational periods of the two planets, again in terms of Earth's period:

$$
\begin{aligned}
& T_{\mathrm{J}} \approx 0.42 T_{\mathrm{E}}, \quad \omega_{\mathrm{J}} \simeq \frac{2 \pi}{0.42}, \\
& T_{\mathrm{N}} \approx 0.8 T_{\mathrm{E}}, \quad \omega_{\mathrm{N}} \simeq \frac{2 \pi}{0.8}
\end{aligned}
$$

With equations (19a)-(19f) we note that $\mathcal{M} \propto L \propto I \omega \propto \omega R^{2}$. Thus, we write

$$
\frac{\mathcal{M}_{\mathrm{N}}}{\mathcal{M}_{\mathrm{J}}} \propto\left(\frac{R_{\mathrm{N}}^{(p)}}{R_{\mathrm{J}}^{(p)}}\right)^{2} \frac{0.42}{0.8} \approx\left(0.35 \times 10^{-3}\right)^{2} 0.53 \gtrsim 1.11 \times 10^{-6}
$$

The latter inequality stems from the fact that this estimate assumes a uniform distribution of charge from the origin to $R_{\mathrm{N}}^{(p)}$, whereas, as noted, the charge is concentrated in the spherical annular domain of the planet. When compared with equation (26b) this rough estimate indicates that the implied magnitude of the magnetic field is within the domain of measured values, thereby corroborating the notion of a liquid metallic hydrogen component of Neptune.

In support of the model that attributes excess radiation to residual heat in planets, a computer model employing the Monte Carlo method for the interior of Neptune (as well as Uranus) was made (Marley et al. 1995). This study is based on a randomly chosen interior constrained by the observed mass, rotation rate, and gravitational moments of the planet. The study leads to a composition gradient of the outer third of the planet and a pressuredensity relation for the central domain consistent with either ice or a mixture of materials with a similar equation of state. It is an alternative model to the present work.

\subsection{Infrared Conversion}

Protons and neutrons emitted in the primary scheme equations (6a) and (6b) in the respective liquid metal regions of Saturn and Jupiter enter the liquid molecular hydrogen domains in which they undergo $(n, p),(p, p)$, and $(t, p)$ interactions. At given energies, $E \leq 3 \mathrm{MeV}$, the $(n, p)$ cross section is $\sigma \leq 5$ barns (McLane et al. 1988), whereas the Coulomb cross section for the remaining two interactions remains finite because of shielding. It follows that the mean free path, $l$, of the product particles is $l \approx$
$1 / \sigma n_{p} \ll \Delta R_{\mathrm{J}, \mathrm{S}}^{L}$, where $\Delta R_{\mathrm{J}, \mathrm{S}}^{L}$ are incremental radii of the liquid hydrogen domains of the respective planets so that protons and neutrons experience a number of collisions in these respective domains. With conservation of energy, this thermalization of particles in the liquid hydrogen domain of the respective planets gives rise to infrared radiation in close agreement with measured values. With the existence of the extended current carrying domain, one may relate the corresponding magnetic field to a dynamo effect. Furthermore, the relatively large inclination of the magnetic field with the rotation axis of the planet is consistent with dynamo currents that lie closer to the planet's surface than to its core (Moore 2000). This property is consistent with the current-loop property of the present work.

Convective storms on the surfaces of giant planets are well established. The question of the relation of such storms to the interior structures of these planets has been examined recently (Hueso et al. 2002; Zang \& Shubert 1966). In the present work it is noted that whereas the respective energy sources of such storms may stem from the planet's interior, it is assumed that such coupling has little effect on the spatial orientation of the proposed D and $\mathrm{H}$ shells of the planets. Recall that the present model includes reasonably well-defined interior shells.

\section{CONCLUSIONS}

An alternative model was described for the excess radiation emitted by the larger planets, each with a liquid metallic hydrogen annular domain about a central ice-rock core.

The present model is based on the attraction between charged bosons that occurs in a charge-neutralizing background at very high pressures. Assuming valid parameters for Jupiter, it is derived that the deuteron density in Saturn is approximately twice that in Jupiter and that particles emitted in reactions in the liquid metal domain are thermalized in the liquid hydrogen domain, resulting in infrared radiation in accord with observed values. The known magnetic moment of Neptune was found to be consistent with positive charge components rotating in a frame of the liquid metallic hydrogen fluid with current density $\approx 8.4 \times 10^{-6} \mathrm{~A} \mathrm{~m}^{-2}$. It was proposed further that the related coupling between current and magnetic field is supported by a dynamo effect. A brief description was included describing the influence on proposed mechanisms and convective storms in the large planets.

I wish to express my appreciation to Steven Seidman and Chung Hoon Lee for assisting me in the preparation of this manuscript. I am equally indebted to Saul Teukolsky, Ira Wasserman, and Edwin Salpeter for alerting me to very pertinent references for this work and to Erick Weinberg, Vaclav Kostroun, Veit Elser, and Bingham Cady for calling my attention to additional concepts in this work.
Baugher, J. F. 1990, The Space-Age Solar System (New York: Wiley)

Blatt, J. M., \& Weisskopf, V. R. 1952, Theoretical Nuclear Physics (New York: Wiley)

Bogoliubov, N. N. 1947, J. Nucl. Phys., 11, 13

Burcham, W. E. 1963, Nuclear Physics (New York: McGraw-Hill)

Coraddu, M., Lissia, M., Messorani, G., \& Quarati, P. 2002, Physica A, 305, 282

de Pater, I., \& Lissauer, J. J. 2001, Planetary Sciences (New York: Cambridge Univ. Press)

Feenberg, E. 1969, Theory of Quantum Fluids (New York: Academic Press)

Foldy, L. L. 1961, Phys. Rev., 124, 649

Friedlander, M. W. 1985, Astronomy (Englewood Cliffs: Prentice Hall)

Girardeau, M. 1962, Phys. Rev., 127, 1809

Goodstein, D. 1975, States of Matter (New York: Dover)

Guillot, T. 1999, Planet. Space Sci., 47, 1183

\section{REFERENCES}

Huang, K. 1987, Statistical Mechanics (2nd ed.; New York: Wiley)

Hubbard, W. B. 1989, in Origin and Evolution of Planetary and Satellite Atmospheres, ed. S. K. Atreya, J. B. Pollack, \& M. S. Matthews (Tucson: Univ. Arizona Press), 539

Hueso, R., Sánchez-Lavega, A., \& Guillot, T. 2002, J. Geophys. Res., 107, E10, 5

Jackson, J. D. 1999, Classical Electrodynamics (New York: Wiley)

Kitamura, H. 2000, ApJ, 539, 888

Leung, W. B., March, N. H., \& Motz, H. 1976, Phys. Lett. A, 56, 425

Liboff, R. L. 1958, Phys. Fluids, 12, 303

1979, Phys. Rev. Lett. A, 74, 323

1994, Phys. Rev. B, 12, 303

2003, Kinetic Theory: Classical, Quantum and Relativistic Descriptions (New York: Springer) 
Mahaffy, P. R., Donahue, T. M., Atreya, S. K., Owen, T. C., \& Niemann, H. B. 1998, Space Sci. Rev., 84, 251

Marley, M. S., Gomez, P., \& Podolak, M. 1995, J. Geophys. Res., 100, 23349

McLane, V., Dunford, C. L., \& Rose, P. F. 1988, Neutron Cross Sections (New York: Academic Press)

Moore, P. 2000, The Data Book of Astronomy (Philadelphia: IOP)

Morrison, D., \& Cruikshank, D. P. 1981, in The New Solar System, ed. J. K. Beaty et al. (New York: Cambridge Univ. Press), 167
Morrison, D., \& Owen, T. 1987, The Planetary System (San Francisco: Addison Wesley)

Ouyed, R., Fundamenski, W. R., Cripps, G. R., \& Sutherland, P. G. 1998, ApJ, 501,367

Salpeter, E., \& Stevenson, D. 1977a, ApJS, 35, 221

1977b, ApJS, 35, 239

Zang, K., \& Shubert, G. 1966, Science, 946, 273 